\title{
Risk of Periodontal Disease: Is there a Correlation with the Type of Antihypertensive Medication?
}

\author{
Scardina $\mathrm{GA}^{1}$, Messina $\mathrm{P}^{1}$ \\ 'Department of Oncology and Surgery Section of Oral Sciences University of Palermo Italy
}

\section{ABSTRACT}

Introduction: The purpose of this study was to evaluate the effects of long-term oral antihypertensive treatment using centrally acting sympatho-inhibitory drugs (clonidine) and beta-blockers (metoprolol) on capillary microcirculation in the labial and periodontal mucosa.

Methods: Sixty subjects were recruited for the study: 20 patients affected by hypertension in treatment with centrally-acting sympatho-inhibitory drugs (64.28 \pm 11.78 years); 20 patients in treatment with beta-blockers (62.03 \pm 9.84 years) and 20 healthy subjects (62.06 \pm 6.72 years). We use the videocapillaroscopic technique to evaluate in vivo the microcirculation of the labial mucosa corresponding to the lower lip and of the periodontal mucosa corresponding to the central superior incisor.

Results: Capillaroscopy revealed a significant increase in the length, diameter, tortuousity and density $(\mathrm{P}<0.05)$ of capillaries in patients affected by hypertension in treatment with centrally acting sympatho-inhibitory drugs, while beta-blockers had a lower effect on the labial and periodontal microcirculation compared to the healthy population.

Conclusions: The capillaroscopic has revealed substantial modifications of the oral microcirculatory pattern in hypertensive patients in treatment with centrally-acting sympatho-inhibitory drugs than with beta-blockers.

Keywords: Hypertension, antihypertensive drug, oral videocapillaroscopy

\section{INTRODUCTION}

Arterial hypertension determines substantial structural modifications at a macro and micro circulatory level ${ }^{1,2}$. In hypertension, a remodelling of the arterial network occurs both from an anatomical and functional point of view. This manifests itself as a reduction of capillary density, a variation of the diameter and an increase in the tone of the vascular wall and in peripheral resistance ${ }^{3,4}$. Considering that the principal role of microcirculation is that of providing for the needs of tissue in terms of the exchange of oxygen and nourishments, these modifications cause the tissue to suffer until damaging the organ ${ }^{5}$. It has been shown how some antihypertensive drugs such as centrallyacting antihypertensive drugs can reduce hypertension and the negative effects correlated to it. The beneficial effects of these categories of medicines are primarily attributed to the lowering of blood pressure, but also to the reduction of the stress caused by oxidization,

\footnotetext{
Correspondence:

Dr. Giuseppe Alessandro Scardina

University of Palermo Section of Oral Sciences

Via Del Vespro, 12990127 Palermo ITALY

Tel : 00390916552209

Email : scardina@odonto.unipa.it
} 
inflammation and endothelial dysfunctions ${ }^{6,7}$. The centrally-acting antihypertensive drugs have a notable effect on vasoreactivity, inducing vasodilatation ${ }^{8}$. The beta-blockers cause vasodilatation, but they have a lower effect on microcirculation ${ }^{9,10}$. Some researchers have demonstrated that the arterial pressure-lowering effect induced by chronic oral antihypertensive treatment with drugs from different pharmacological classes is not always accompanied by the same reversion in functional and structural capillary rarefaction.

The aim of our study was to evaluate the effects of oral long-term antihypertensive treatment using centrallyacting sympatho-inhibitory drugs and beta-blockers on capillary microcirculation in the labial and periodontal mucosa.

\section{METHODS}

The study population comprised of sixty subjects. Twenty patients affected by hypertension under treatment with centrally-acting sympatho-inhibitory drugs (clonidine $300 \mathrm{mg} 2$ times for 3 years) (10 males and 10 females; $64.28 \pm 11.78$ years) were in the first group. Twenty patients affected by hypertension under treatment with beta-blockers (metoprolol 100 mg 2 times for 3 years) $(10$ males and 10 females; $62.03 \pm 9.84$ years) were in the second group. Twenty age-and-sex matched healthy subjects $(10$ males and 10 females; $62.06 \pm 6.72$ years) were enrolled as controls.

At the moment of the videocapillaroscopic examination , the blood pressure of the study subjects was well controlled. All subjects recruited in the control group did not have any systemic pathologies. They took no drugs that could influence oral microcirculation and they were non-smokers. After having signed the informed consent form allowing the researchers to proceed with the videocapillaroscopic examination and the use of personal data, the patients were submitted to a complete study of labial microcirculation corresponding to the lower lip (frenulum) surface and of periodontal microcirculation corresponding to the central superior incisor. All the subjects enrolled in the study had an index of visible plaque on the surface of the teeth that was equal to 0 . The control and case groups were analysed in the same phases and in the same conditions to avoid that the results could be conditioned by phases, position and breeze in the room. The study was approved by the ethical committee of the University of Palermo.

\section{Videocapillaroscopy}

Oral videocapillaroscopy is a simple, reproducible, non- invasive, panoramic technique that is performed in vivo and is well-tolerated by the patient. This computerized technique is used with specific software
(DS Medigroup, Milan Italia) that allows the acquisition and the elaboration of the data. The videocapillaroscope is made up of a central unit, a fibre optic probe with a video-optic terminal and a high resolution colour monitor. The lens has a focal spot of $1.811 \mathrm{~mm}$ and enlargements which vary from 10 to $1000 X$. For the study in question, a 200X enlargement was used. The morphofunctional evaluation of microcirculation is based on specific parameters: length of the capillary loop, diameter of the loop, diameter of afferent and efferent loops, capillary tortuousness and density. The videocapillaroscopic investigation was always performed with the same light source, at the same room temperature $\left(23^{\circ} \mathrm{C}\right)$, in the morning, with the same operator (GAS) and repeated two times for every area under investigation ${ }^{11}$.

The following static parameters were analysed:

\section{Non-Parametric data:}

- Capillary loop visibility (graded 1 to 4): (1) simple focusing - within 30s from the beginning of the examination; (2) average focusing - over 30 s and within 2 min; (3) difficult focusing - over $2 \mathrm{~min}$; (4) impossible focusing;

- Orientation to the surface (graded $A, B$ or $A B$ ): (A) capillary loop course parallel to the surface; (B) capillary loop course perpendicular to the surface; $(A B)$ both parallel and perpendicular;

- Microhemorrhages (graded 0 or 1): (0) absence; (1) presence;

- Characteristics of the capillary loops (marks 0 or 1): (0) absence; (1) presence.

\section{Parametric data:}

- Capillary length;

- Capillary density (number of visible capillary loops per square millimetre - value obtained from the average of the two observations for examined area);

- Capillary loop diameter (total, afferent and efferent loop);

- Tortuosity: number of crossings in the capillary loop (value obtained from the average of the two observations for the examined area).

\section{Statistic analysis}

Statistic analysis was conducted using the MannWhitney $U$ test. The significance level was of $P<0.05$. The collected data was analyzed with StatView 5.0.1/ SAS Institute Inc., Cary, NC). The acquired data refer to the mean value among those acquired in the measurements for every pre-established parameter. 


\section{RESULTS}

In all the cases and during all the phases of the study, the visibility of microcirculation during the capillaroscopic examination was clear and focussing simple and immediate. The direction of the capillaries of the labial mucosa was parallel to the surface (score A); the orientation of the microcirculation corresponding to the periodontal mucosa was perpendicular to the surface (score B).

In the healthy subjects and the control groups, the visibility of the capillary loops was clear. No microhaemorrhage was observed in the case and the control groups (Figures. 1,2,3). The statistic analysis was conducted comparing the mean values of the measurements.

Table 1 shows the observed value for capillary length, loop diameter, density and tortousity of the vessels, diameter of afferent and efferent loops and periodontal density. Beside the diameter of afferent and efferent loops, all the parameters showed statistical significance.

Table 1. Oral microvascular differences in patients affected by hypertension in treatment with clonidine (group A) and metaprololol (group B)

\begin{tabular}{|l|l|l|l|l|}
\hline Examined Parameter & $\begin{array}{l}\text { Healthy subjects } \\
(\mathrm{n}=20)\end{array}$ & Group A ( $=20)$ & Group B ( $=20)$ & $\begin{array}{l}\text { P value } \\
\text { (Mann Whitney Test) }\end{array}$ \\
\hline Capillary length & $0.26 \pm 0.96$ & $0.55 \pm 0.96$ & $0.33 \pm 0.096$ & 0.000000067 \\
\hline Diameter loop & $0.04 \pm 0.010$ & $0.52 \pm 0.006$ & $0.35 \pm 0.01$ & 0.00000006719 \\
\hline Density & $27.75 \pm 4.788$ & $59.05 \pm 7.74$ & $34.05 \pm 4.22$ & 0.0000006796 \\
\hline Tortuosity & $0.789 \pm 0.402$ & $3.594 \pm 0.096$ & $2.33 \pm 0.08$ & 0.0000329 \\
\hline Diameter Afferent loop & $0.012 \pm 0.007$ & $0.018 \pm 0.002$ & $0.013 \pm 0.007$ & 0.02358 \\
\hline Diameter efferent loop & $0.015 \pm 0.003$ & $0.024 \pm 0.0036$ & $0.014 \pm 0.0036$ & 0.0000004642 \\
\hline Periodontal density & $18.45 \pm 0.02$ & $42.23 \pm 0.23$ & $29.17 \pm 0.14$ & 0.00000067 \\
\hline
\end{tabular}

Group A, Clonidine group; group B, Metoprolol group. All the valuses are expressed in mean \pm SD

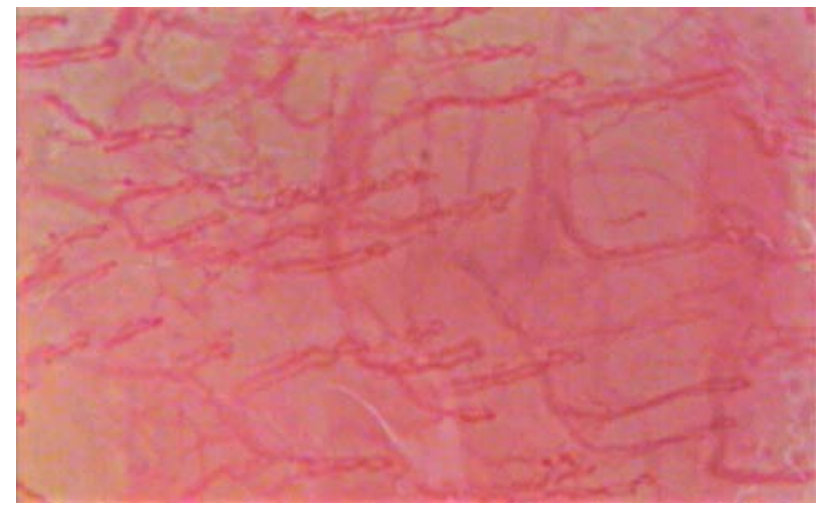

Figure 1. Oral microcirculation of patients affected by hypertension in treatment with antihypertensive drugs. A picture particularly dense in capillaries alternating "forked" capillaries and tortuous ones. (magnification 200X

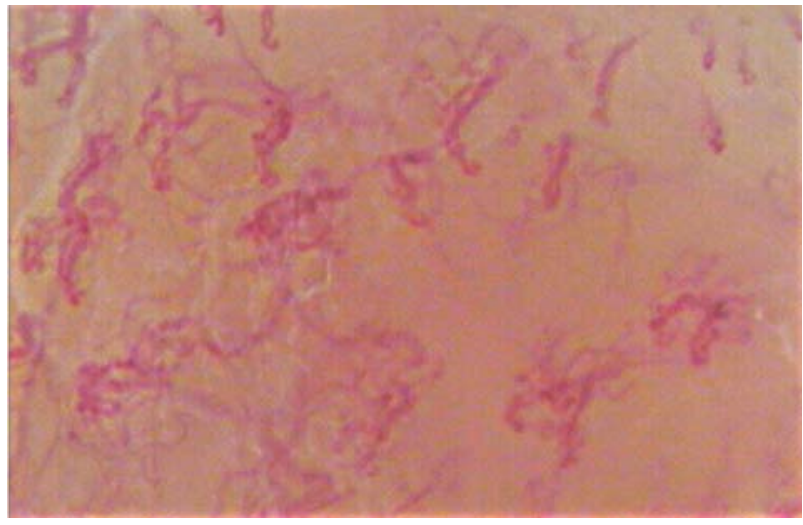

Figure 2. Oral microcirculation of patients affected by hypertension in treatment with antihypertensive drugs. Capillaroscopic picture which shows tangled capillaries that seem very tortuous, indistinguishable and overlapping. (magnification 200X) 


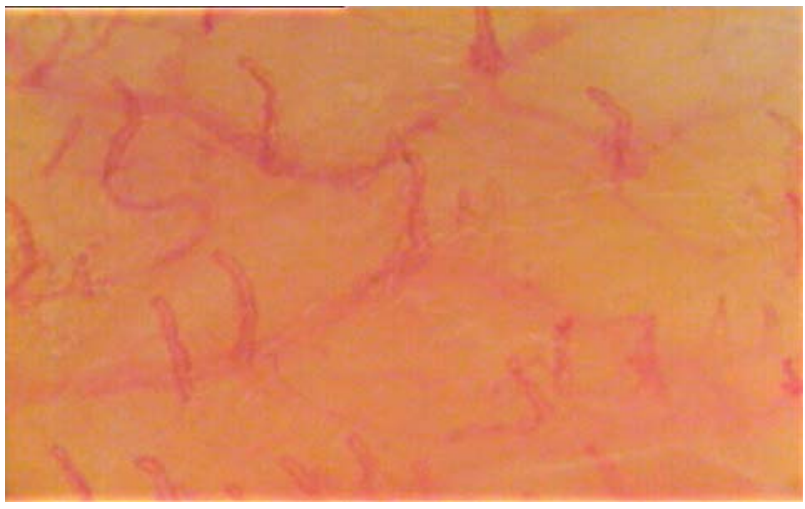

Figure 3. Oral microcirculation of healthy subject. (magnification 200X)

\section{DISCUSSION}

Drug-induced periodontitis, as a well-documented side effect, has been reported with the systemic use of antihypertensive drugs. In our study we evaluated the effect of different antihypertensive drugs on capillary microcirculation in the labial and periodontal mucosa. We also evaluated if there exist a correlation between the types of antihypertensive drug, the characteristic of microcirculation and the eventual risk of periodontitis.

A 2009 study published data produced by the investigation of the ocular fundus in hypertensive patients who were taking amlodipine. Through photographs of the capillaries of the retina, they found an alteration of the length and the diameter of the capillaries which could be justified by the vasodilatory effect of the drug $^{12}$. Research carried out using the laser Doppler flowmetry detected the same alteration and proposed a treatment without the use of medicines, based instead on the use of magnetotherapy ${ }^{13}$. The reduction of vascular rarefaction following treatment with ACE inhibitors, beta blockers and other antihypertensive drugs has also been tested on mice through the use of video microscopy and the study of histological samples. The results of this study were different depending on the molecules taken by the group of cases composed of rats with spontaneous hypertension. Atenolol did not induce any change in functional capillary density in the hypertensive rats. Losartan and nifedipine completely reversed functional capillary rarefaction in both muscle and cutaneous tissues, whereas enalapril significantly increased functional capillary density only in the skin.

These results suggest that different pharmacologic classes of antihypertensive drugs with similar effect on blood pressure differ in terms of their effect on the microcirculation ${ }^{14}$. The same authors have conducted similar studies on rats using centrally-acting sympathoinhibitory drugs. In this case, the results showed that long-term antihypertensive treatment with centrally-acting drugs enhanced tissue perfusion and reversed capillary rarefaction in the skeletal muscle of spontaneously hypertensive rats ${ }^{15}$. Instead, Penna et al. conducted studies using nail fold videocapillaroscopy. The obtained results sustain that there are no modification of vascular alterations in hypertensive subjects treated pharmacologically ${ }^{16}$.

There exists only one study in literature regarding oral microcirculation and it was conducted in the sublingual region using orthogonal polarized spectral imaging and sidestream darkfield-techniques on patients with a history of recent cardiogenic shock.

This study reports an impairment of microcirculation with organ hypoperfusion. These authors observed that cardiac output is correlated to disturbance in microflow in the smallest vessels and proposed that the on-line evaluation of microcirculation in vivo may be a valid tool for optimizing therapeutic measures in high risk patients ${ }^{17}$.

In our research we observed that oral capillaroscopy allows an investigation of oral microcirculation in an immediate and non-invasive way. The capillaroscopic study consists of an evaluation of the parametric and non-parametric data, which are statistically analyzed, and of a morphological study of the capillaroscopic picture. The statistical investigation has shown a significant increase in the length, diameter, tortuousity and labial and periodontal density of the capillaries. We observed a different increase of the oral microcirculatory parameter according to the antihypertensive drug used. The results obtained appear in line with those found in literature for what concerns the increase of the capillary diameter and density. The data on the length seems controversial. The increase in tortuosity and capillary length appears compatible, however, with the vasodilatation and the vasoreactivity induced by the administered medicines.

In some cases, the capillaries took on a "woven" aspect, revealing themselves to be extended and parallel to one another. The density had already been evident in an earlier examination, during the capillaroscopic procedure. The increase of the length, of the capillary diameter and the particular disposition of the intracapillary cross as well as of the recurrent "tangled" image are presumably the expression of the vasodilatory effect of the drugs taken by the patients recruited in the study. 
The capillaroscopic study has underlined substantial modifications of the microcirculatory pattern in hypertensive patients taking antihypertensive medicines. This could be traced back to the positive effects of such medicines on the macro and microcirculation and to the remodelling that they produce on the vascular pattern.

\section{CONCLUSIONS}

The results of the present study suggest that centrallyacting sympatho-inhibitory drugs have a more significant effect on labial and periodontal microcirculation than do beta-blockers. The approach in the control of periodontal microcirculation and periodontitis correlated to drugs should be substitution of the drug. A change in medication should only be considered for those patients for whom the new medication can offer some advantage for control of their hypertension and who are at high risk for periodontitis.

\section{REFERENCES}

1. Agabiti-Rosei E. From macro-to microcirculation: benefits in hypertension and diabetes. J Hypertens Suppl. 2008;26:S15-9.

2. Dabiri Askari A, Feihl F, Waeber B. Arterial hypertension: macrocirculation and microcirculation. Rev Med Suisse. 2009;5:1778-82.

3. Feihl F, Liaudet L, Waeber B. The macrocirculation and microcirculation of hypertension. Curr Hypertens Rep. 2009;11:182-9.

4. Vicaut E. Hypertension and the microcirculation. Arch Mal Coeur Vaiss. 2003;96:893-903.

5. Mourad JJ, Laville M. Is hypertension a tissue perfusion disorder? Implications for renal and myocardial perfusion. J Hypertens Suppl. 2006;24:S10-6.

6. Ishida $\mathrm{Y}$, Tomori $\mathrm{K}$, Nakamoto H, Imai H, Suzuki H. Effects of antihypertensive drugs on peritoneal vessels in hypertensive dogs with mild renal insufficiency. Adv Perit Dial. 2003;19:10-4.

7. Cohuet G, Struijker-Boudier H. Mechanisms of target organ damage caused by hypertension: therapeutic potential. Pharmacol Ther. 2006;111:81-98.

8. Versari D, Virdis A, Ghiadoni L, Daghini E, Duranti E, Masi S, et al. Effect of verapamil, trandolapril and their combination on vascular function and structure in essential hypertensive patients. Atherosclerosis. 2009;205:214-20.

9. Zanchetti A. Clinical pharmacodynamics of nebivolol: new evidence of nitric oxide-mediated vasodilating activity and peculiar haemodynamic properties in hypertensive patients. Blood Press Suppl. 2004;1:17-32.
10. Kamp O, Metra M, Bugatti S, Bettari L, Dei Cas A, Petrini $\mathrm{N}$, et al. Nebivolol: haemodynamic effects and clinical significance of combined beta-blockade and nitric oxide release. Drugs. 2010;70:41-56.

11. Scardina GA, Ruggieri A, Messina P. Oral microcirculation observed in vivo by videocapillaroscopy: a review. J Oral Sci. 2009;51:1-10.

12. Thom S, Stettler C, Stanton A, Witt N, Tapp R, Chaturvedi $\mathrm{N}$, et al. Differential effects of antihypertensive treatment on the retinal microcirculation: an anglo-scandinavian cardiac outcomes trial substudy. Hypertension. 2009;54:405-408.

13. Kul'chitskaia DB. Rehabilitative medical technology for the correction of microcirculatory disturbances in patients with arterial hypertension. Vopr Kurortol Fizioter Lech Fiz Kult. 2009;5:9-11.

14. Sabino B, Lessa MA, Nascimento AR, Rodrigues CAHenriques MG, Garzoni LR et al. Effects of antihypertensive drugs on capillary rarefaction in spontaneously hypertensive rats: intravital microscopy and histologic analysis. J Cardiovasc Pharmacol. 2008;51:402-9.

15. Nascimento AR, Lessa MA, Sabino B, Bousquet $P$, Tibirica E. Microvascular effects of centrally-acting antihypertensive drugs in spontaneously hypertensive rats. J Cardiovasc Pharmacol. 2010;55:204-7.

16. Penna GL, Garbero Rde F, Neves MF, Oigman W, Bottino DA, Bouskela E. Treatment of essential hypertension does not normalize capillary rarefaction. Clinics (Sao Paulo). 2008;63:613-8.

17. Jung C, Ferrari M, Rodiger C, Fritzenwanger M, Goebel B, Lauten A, et al. Evaluation of the sublingual microcirculation in cardiogenic shock Clin Hemorheol Microcirc. 2009;42:1418. 